DOI: $10.15393 / \mathrm{j} 3$.art.2018.4310

UDC 517.51, 517.52, 517.58

EDWARD NEUMAN

\title{
GENERALIZED GUDERMANNIAN FUNCTION
}

\begin{abstract}
Wilker and Huygens-type inequalities involving generalized Gudermannian function and its inverse function are established. These results are obtained with the aid of the p-version of the Schwab-Borchardt mean. Generalized one-parameter trigonometric and hyperbolic functions play a crucial role in this paper.
\end{abstract}

Key words: Generalized Gudermannian function and its inverse function, generalized trigonometric and generalized hyperbolic functions, Gauss hypergeometric function, p-version of the Schwab-Borchardt mean, inequalities

2010 Mathematical Subject Classification: 26E60, 26D07, 26D20

1. Introduction. The Gudermannian function and its inverse function, denoted by $g d$ and $g d^{-1}$, respectively, are given by [16, 4.23 (viii)]

$$
g d(x)=\int_{0}^{x} \frac{1}{\cosh t} d t, \quad x \in \mathbb{R}
$$

and

$$
g d^{-1}(x)=\int_{0}^{x} \frac{1}{\cos t} d t, \quad|x|<\pi / 2 .
$$

It is known (see, e.g., [16, 4.23 (viii)]) that

$$
g d(x)=\sin ^{-1}(\tanh x)=\cos ^{-1}(\operatorname{sech} x)=\tan ^{-1}(\sinh x)
$$

and

$$
g d^{-1}(x)=\sinh ^{-1}(\tan x)=\cosh ^{-1}(\sec x)=\tanh ^{-1}(\sin x) .
$$

(C) Petrozavodsk State University, 2018 
Functions $g d$ and $g d^{-1}$ relate circular and hyperbolic functions without using complex numbers.

One of the goals of this paper is to define and study generalizations of $g d$ and $g d^{-1}$. Among the mathematical tools used throughout the sequel is the p-version of the Schwab-Borchardt mean introduced and investigated by this author in [11, 12. Some known results involving this mean are also included in Section 2. Definitions of the generalized Gudermannian function and its inverse function are provided in Section 3. The lower and upper bounds for these functions are established in Section 4. The Wilker and Huygens-type inequalities involving generalized Gudermannian function $g d_{p}$ and its inverse function $g d_{p}^{-1}$ are obtained in Section 5 . Here and in what follows letter $p$ stands for a parameter which is always greater than 1.

2. Definitions and preliminary results. The generalized trigonometric functions have been introduced by P. Lindqvist in [7]. It is known that they are eigenfunctions of the Dirichlet problem for the one-dimensional $p$-Laplacian. They are also useful in the quasi-conformal theory, geometric function theory and in the investigations of the Ramanujan modular equations. For more details concerning generalized trigonometric functions, generalized hyperbolic functions, and inequalities involving these functions the interested reader is referred to [2]-[7, [9]-[11, [13].

For the reader's convenience we recall first definition of the celebrated Gauss hypergeometric function $F(a, b ; c ; z)$ :

$$
F(a, b ; c ; z)=\sum_{n=0}^{\infty} \frac{(a, n)(b, n)}{(c, n)} \frac{z^{n}}{n !}, \quad|z|<1,
$$

where $(a, n)=a(a+1) \ldots(a+n-1)(n \neq 0)$ is the shifted factorial or Appell symbol, with $(a, 0)=1$ if $a \neq 0$, and $c \neq 0,-1,-2, \ldots$

In the sequel we shall adopt notation and definitions used in [4. Let

$$
\pi_{p}=2 \frac{\pi / p}{\sin (\pi / p)} .
$$

Further, let

$$
a_{p}=\frac{\pi_{p}}{2}, b_{p}=2^{-1 / p} F\left(\frac{1}{p}, \frac{1}{p} ; 1+\frac{1}{p} ; \frac{1}{2}\right), c_{p}=2^{-1 / p} F\left(1, \frac{1}{p} ; 1+\frac{1}{p} ; \frac{1}{2}\right) .
$$

Also, let $I=(0,1)$ and let $J=(1, \infty)$. The generalized trigonometric and 
hyperbolic functions utilized in this paper are the following homeomorphisms

$$
\sin _{p}:\left(0, a_{p}\right) \rightarrow I, \quad \cos _{p}:\left(0, a_{p}\right) \rightarrow I, \quad \tan _{p}:\left(0, b_{p}\right) \rightarrow I
$$

and

$$
\sinh _{p}:\left(0, c_{p}\right) \rightarrow I, \quad \cosh _{p}:(0, \infty) \rightarrow J, \quad \tanh _{p}:(0, \infty) \rightarrow I .
$$

The inverse functions of $\sin _{p}$ and $\sinh _{p}$ can be represented as follows [6]:

$$
\begin{aligned}
\sin _{p}^{-1} u & =\int_{0}^{u}\left(1-t^{p}\right)^{-1 / p} d t=u F\left(\frac{1}{p}, \frac{1}{p} ; 1+\frac{1}{p} ; u^{p}\right), \\
\sinh _{p}^{-1} u & =\int_{0}^{u}\left(1+t^{p}\right)^{-1 / p} d t=u F\left(\frac{1}{p}, \frac{1}{p} ; 1+\frac{1}{p} ;-u^{p}\right),
\end{aligned}
$$

where $u \in I$.

The last two functions are extended to negative arguments as follows (see, e.g., [6]):

$$
\sinh _{p}^{-1} u=-\sinh _{p}^{-1}(-u), \quad u \in(-1,0) .
$$

Function $\sin _{p} u$ is extended to negative values of its argument in a similar way. We omit further details.

The inverse functions of $\cos _{p}, \cosh _{p}, \tan _{p}$, and $\tanh _{p}$ can be expressed in terms of $\sin _{p}^{-1}$ and $\sinh _{p}^{-1}$. We have

$$
\begin{aligned}
\cos _{p}^{-1} u & =\sin _{p}^{-1}\left(\sqrt[p]{1-u^{p}}\right) \\
\cosh _{p}^{-1} u & =\sinh _{p}^{-1}\left(\sqrt[p]{u^{p}-1}\right) \\
\tan _{p}^{-1} u & =\sin _{p}^{-1}\left(\frac{u}{\sqrt[p]{1+u^{p}}}\right)
\end{aligned}
$$

and

$$
\tanh _{p}^{-1} u=\sinh _{p}^{-1}\left(\frac{u}{\sqrt[p]{1-u^{p}}}\right)
$$


For later use let us record some known definitions and formulas. Let us begin with the definition of $p$-version of the function cosine. We follow [5] to define

$$
\cos _{p} x=\frac{d}{d x} \sin _{p} x
$$

It follows from (7) that

$$
\left|\cos _{p} x\right|^{p}+\left|\sin _{p} x\right|^{p}=1, \quad x \in \mathbb{R} .
$$

Corresponding formulas for the $p$-hyperbolic functions read as follows [3, 5]:

$$
\cosh _{p} x=\frac{d}{d x} \sinh _{p} x
$$

and

$$
\left|\cosh _{p} x\right|^{p}-\left|\sinh _{p} x\right|^{p}=1, \quad x \in \mathbb{R} .
$$

We shall also utilize functions

$$
\tan _{p} x=\frac{\sin _{p} x}{\cos _{p} x}
$$

where $x \in \mathbb{R}$ and $x \neq(2 k+1) a_{k}, k \in \mathbb{Z}$, and

$$
\tanh _{p} x=\frac{\sinh _{p} x}{\cosh _{p} x}
$$

(see, e.g., [3, 5]). It is known (see [6]) that

$$
\frac{d}{d x} \tan _{p}(x)=1+\left|\tan _{p}(x)\right|^{p}, \quad \frac{d}{d x} \tanh _{p}(x)=1-\left|\tanh _{p}(x)\right|^{p} .
$$

The p-version of the Schwab-Borchardt mean of two numbers $a \geq 0$ and $b>0$, denoted by $S B_{p}(a, b) \equiv S B_{p}$, is defined as

$$
S B(a, b)= \begin{cases}\frac{\sqrt[p]{b^{p}-a^{p}}}{\cos _{p}^{-1}(a / b)}, & 0 \leq a<b \\ \frac{\sqrt[p]{a^{p}-b^{p}}}{\cosh _{p}^{-1}(a / b)}, & b<a \\ a, & a=b\end{cases}
$$


(see [11, (22)], [12, (8)]). It follows from 18$)$ that $S B_{p}(a, b)$ is not symmetric in its arguments and is a homogeneous function of degree 1 in $a$ and $b$. This mean has been studied extensively in [11, 12].

For the reader's convenience we include below some results which will be used in the sequel. We begin with the following one which has been established in [11.

Let the positive numbers $a$ and $b$ be such that $a>b$. Then

$$
S B_{p}(a, b)<S B_{p}(b, a) .
$$

Inequality (19) is reversed if $a<b$.

For later use let us recall the following result [11]. Let $\alpha=1 / p$ and $\gamma=1 /(p+1)$. Then the inequality

$$
a^{\gamma} b^{1-\gamma}<\left[S B_{p}(b, a)\right]^{\alpha} b^{1-\alpha}<S B_{p}(a, b)
$$

holds true for positive and unequal numbers $a$ and $b$. Moreover, if $a>b$, then

$$
S B_{p}(a, b)<\sigma a+(1-\sigma) b
$$

where

$$
\sigma= \begin{cases}\frac{1}{p+1}, & 1<p \leq 2 \\ \frac{1}{3}, & p \geq 2\end{cases}
$$

(see [12]). Finally, the following result

$$
S B_{p}(a, b)<\left(\gamma a^{p}+(1-\gamma) b^{p}\right)^{1 / p}
$$

is established in [12. Here $\gamma$ is the same as in (20).

We close this section with three results which appear in mathematical literature. The first one reads as follows [14]:

Theorem 1. Let $f$ be a positive and strictly monotonic function defined on the subset $D$ of the positive semiaxis. Assume that $f^{-1}$ is strictly increasing and also that $f(x) \geq x(f(x) \leq x)$ for all $x \in D$. If the function $f(x) / x$ is increasing (decreasing) on $D$, then

$$
\frac{f(x)}{x} \geq \frac{x}{f^{-1}(x)}
$$


Inequality 23 is reversed if the function $f(x) / x$ is decreasing (increasing) on $D$.

We shall employ the following theorem (see [1]):

Theorem 2. Let the functions $f$ and $g$ be continuous on $[c, d]$, differentiable on $(c, d)$, and such that $g^{\prime}(t) \neq 0$ on $(c, d)$. If $\frac{f^{\prime}(t)}{g^{\prime}(t)}$ is (strictly) increasing (decreasing) on $(c, d)$, then the functions $\frac{f(t)-f(d)}{g(t)-g(d)}$ and $\frac{f(t)-f(c)}{g(t)-g(c)}$ are also (strictly) increasing (decreasing) on $(c, d)$.

The next theorem will be utilized in the subsequent sections of this paper:

Theorem 3. Let $u, v, \alpha, \beta, \gamma$ and $\delta$ be positive numbers which satisfy the following conditions

(i) $\min (u, v)<1<\max (u, v)$,

(ii) $1<u^{\gamma} v^{\delta}$.

Then the inequality

$$
\alpha+\beta<\alpha u^{r}+\beta v^{s}
$$

is satisfied provided $u<1<v$ and

$$
s>0 \quad \text { and } \quad r \alpha \delta \leq s \beta \gamma .
$$

If in addition the positive numbers $u, v, \gamma$ and $\delta$ are such that

(iii) $\gamma+\delta<\gamma \frac{1}{u}+\delta \frac{1}{v}$,

then the inequality (24) is satisfied if

$$
r \leq s \leq-1 \quad \text { and } \quad \beta \gamma \leq \alpha \delta
$$

(see [8], Theorem 3.1).

Conditions of validity of (24) when $v<1<u$ are also obtained in [8]. The counterpart of 25 is

$$
r>0 \quad \text { and } \quad s \beta \gamma \leq r \alpha \delta
$$

while the conditions 26 are replaced now by

$$
s \leq r \leq-1 \quad \text { and } \quad \alpha \delta \leq \beta \gamma .
$$


3. Generalized Gudermannian function and its inverse function. Generalizations of formulas (1) - (4) are given in this section. Definition of the generalized Gudermannian function reads as follows:

$$
g d_{p}(x)=\int_{0}^{x} \frac{1}{\left(\cosh _{p} t\right)^{p-1}} d t, \quad x \in \mathbb{R} .
$$

Since $g d_{p}(x)$ is the odd function we may assume without loss of generality that $x>0$.

It is easy to show that the function $g d_{p}$ can be expressed in terms of the generalized circular and hyperbolic functions. We have

Lemma 1. The following formulas

$$
g d_{p}(x)=\sin _{p}^{-1}\left(\tanh _{p} x\right)=\tan _{p}^{-1}\left(\sinh _{p} x\right)
$$

are valid.

Proof. We begin by showing that the first part of $(30)$ holds true. To this aim we differentiate, using (5), (14), (16), and (29), to obtain

$$
\frac{d}{d x} \sin _{p}^{-1}\left(\tanh _{p} x\right)=\left(1-\tanh _{p}^{p} x\right)^{1-1 / p}=\left(\cosh _{p} x\right)^{1-p}=\frac{d}{d x} g d_{p} x .
$$

For the proof of the second equality in $(30)$ we utilize formulas (9), (14) and (16) with $u=\sinh _{p} x$ to obtain

$$
\begin{array}{r}
\tan _{p}^{-1}\left(\sinh _{p} x\right)=\sin _{p}^{-1}\left(\frac{\sinh _{p} x}{\sqrt[p]{1+\sinh _{p}^{p} x}}\right)= \\
=\sin _{p}^{-1}\left(\frac{\sinh _{p} x}{\cosh _{p} x}\right)=\sin _{p}^{-1}\left(\tanh _{p} x\right) .
\end{array}
$$

This completes the proof.

We will deal now with the inverse function of $g d_{p}$.

Lemma 2. The inverse function of the generalized Gudermannian function is

$$
g d_{p}^{-1}(x)=\int_{0}^{x} \frac{1}{\left(\cos _{p} t\right)^{p-1}} d t, \quad|x|<a_{p} .
$$


Moreover,

$$
g d_{p}^{-1}(x)=\sinh _{p}^{-1}\left(\tan _{p} x\right)=\tanh _{p}^{-1}\left(\sin _{p} x\right) .
$$

Proof. We begin by showing that the first equality in $(32)$ is valid. Differentiation with the use of (6), (17), 15), 12), and (31) yields

$$
\frac{d}{d x} \sinh _{p}^{-1}\left(\tan _{p} x\right)=\left(1+\tan _{p}^{p} x\right)^{1-1 / p}=\left(\cos _{p} x\right)^{1-p}=\frac{d}{d x} g d_{p}^{-1} x .
$$

For the proof of the second equality in 32 we utilize formulas 10 with $u=\sin _{p} x, 12$ and 15 to obtain

$$
\begin{array}{r}
\tanh _{p}^{-1}\left(\sin _{p} x\right)=\sinh _{p}^{-1}\left(\frac{\sin _{p} x}{\sqrt[p]{1-\sin _{p}^{p} x}}\right)= \\
=\sinh _{p}^{-1}\left(\frac{\sin _{p} x}{\cos _{p} x}\right)=\sinh _{p}^{-1}\left(\tan _{p} x\right) .
\end{array}
$$

To complete the proof we have to show that

$$
g d_{p}\left(g d_{p}^{-1}(x)\right)=g d_{p}^{-1}\left(g d_{p}(x)\right)=x
$$

holds for all $-a_{p}<x<a_{p}$. This an elementary task which requires application of formulas (30) and (32). We leave it to the interested reader.

4. Bounds for functions $g d_{p}$ and $g d_{p}^{-1}$. The goal of this section is to derive lower and upper bounds for two functions introduced in the previous section. Bounding expressions depend on the one-parameter generalized trigonometric and hyperbolic functions and their inverse functions. In the proofs of the main results in Section 5 we shall often appeal to the following

Lemma 3. The following formulas

$$
\begin{aligned}
S B_{p}\left(1, \cos _{p} t\right)=\frac{\sin _{p} t}{g d_{p}^{-1}(t)}, & t \in\left(0, a_{p}\right), \\
S B_{p}\left(1, \cosh _{p} t\right)=\frac{\sinh _{p} t}{g d_{p}(t)}, & t \in\left(0, c_{p}\right), \\
S B_{p}\left(\cos _{p} t, 1\right)=\frac{\sin _{p} t}{t}, & t \in\left(0, a_{p}\right), \\
S B_{p}\left(\cosh _{p} t, 1\right)=\frac{\sinh _{p} t}{t}, & t \in\left(0, c_{p}\right),
\end{aligned}
$$


are valid.

Proof. Formulas (35) and (36) are established in [10]. In order to prove formula (33) we utilize (18) with $a=1$ and $b=\cos _{p} t$ followed by application of (12), (8), (15), and (32). This yields

$$
\begin{aligned}
S B_{p}\left(1, \cos _{p} t\right)=\frac{\sqrt[p]{1-\cos _{p}^{p} t}}{\cosh _{p}^{-1}\left(1 / \cos _{p} t\right)} & =\frac{\sin _{p} t}{\sinh _{p}^{-1}\left(\sqrt[p]{1 / \cos _{p}^{p} t-1}\right)}= \\
& =\frac{\sin _{p} t}{\sinh _{p}^{-1}\left(\tan _{p} t\right)}=\frac{\sin _{p} t}{g d_{p}^{-1}(t)} .
\end{aligned}
$$

For the proof of (34) we apply (18) with $a=1$ and $b=\cosh _{p} t$ together with the use of formulas (14), (7), (16) and (30). We have

$$
\begin{aligned}
S B_{p}\left(1, \cosh _{p} t\right)=\frac{\sqrt[p]{\cosh _{p}^{p} t-1}}{\cos _{p}^{-1}\left(1 / \cosh _{p} t\right)} & =\frac{\sinh _{p} t}{\sin _{p}^{-1}\left(\sqrt[p]{\left.1-1 / \cosh _{p}^{p} t\right)}\right.}= \\
& =\frac{\sinh _{p} t}{\sin _{p}^{-1}\left(\tanh _{p} t\right)}=\frac{\sinh _{p} t}{g d_{p}(t)} .
\end{aligned}
$$

The proof is complete.

Multiplying both sides of (35) and (33) by $1 / \cos _{p} t$ and utilizing homogeneity of the Schwab-Borchardt mean $S B_{p}$ we obtain two formulas

$$
S B_{p}\left(1, \frac{1}{\cos _{p} t}\right)=\frac{\tan _{p} t}{t}, \quad t \in\left(0, a_{p}\right),
$$

and

$$
S B_{p}\left(\frac{1}{\cos _{p} t}, 1\right)=\frac{\tan _{p} t}{g d_{p}^{-1}(t)}, \quad t \in\left(0, a_{p}\right) .
$$

We are in a position to prove the following:

Theorem 4. Let $t \in\left(0, c_{p}\right)$. Then

$$
\left(\frac{\sinh _{p} t}{t}\right)^{1-p}<\frac{g d_{p}(t)}{t}<\frac{\sinh _{p} t}{t}\left(\cosh _{p} t\right)^{-p /(p+1)} .
$$

If $t \in\left(0, a_{p}\right)$, then

$$
\left(\frac{\sin _{p} t}{t}\right)^{1-p}<\frac{g d_{p}^{-1}(t)}{t}<\frac{\sin _{p} t}{t}\left(\cos _{p} t\right)^{-p /(p+1)} .
$$


Proof. The two-sided inequality (39) can be obtained by use of 20 with $a=\cosh _{p} t$ and $b=1$ followed by application of (34) and (36) to obtain

$$
\left(\cosh _{p} t\right)^{1 /(p+1)}<\left(\frac{\sinh _{p} t}{g d_{p}(t)}\right)^{1 / p}<\frac{\sinh _{p} t}{t}
$$

A little algebra yields the assertion. Inequalities 40 can be derived in a similar manner. We use (20) with $a=\cos _{p} t$ and $b=1$ and next utilize (33) and (35) to obtain

$$
\left(\cos _{p} t\right)^{1 /(p+1)}<\left(\frac{\sin _{p} t}{g d_{p}^{-1}(t)}\right)^{1 / p}<\frac{\sin _{p} t}{t} .
$$

Easy computations yield the desired inequalities 40 .

We close this section with the following

Theorem 5. Let $\sigma$ be the same as in 21). If $t \in\left(0, a_{p}\right)$, then

$$
\frac{\sin _{p} t}{\sigma+(1-\sigma) \cos _{p} t}<g d_{p}^{-1}(t)<t\left(\frac{\tan _{p} t}{t}\right)^{1-1 / p} .
$$

Proof. The left hand-side inequality in (41) follows from (21) provided $a=1$ and $b=\cos _{p} t$. Making use of (33) we get

$$
\frac{\sin _{p} t}{g d_{p}^{-1}(t)}<\sigma+(1-\sigma) \cos _{p} t
$$

For the proof of the right-hand side inequality in (41) we employ the second and third members of 20 with $a$ and $b$ as above. This yields

$$
\left(\frac{\sin _{p} t}{t} \cos _{p}^{p-1} t\right)^{1 / p}<\frac{\sin _{p} t}{g d_{p}^{-1}(t)} .
$$

The assertion now follows. In the last step we have utilized formulas (35) and (33). The proof is complete.

5. Main results. In this section we derive five Wilker and Huygenstype inequalities involving two major functions studied in this paper. Some of these results, when $p=2$, reduce to those obtained earlier by this author and published in [15]. 
For the compactness of presentation letters $\alpha$ and $\beta$ will always stand for positive numbers.

We begin this section with the following

Theorem 6. The inequality

$$
\alpha+\beta<\alpha\left(\frac{g d_{p}(x)}{x}\right)^{r}+\beta\left(\frac{g d_{p}^{-1}(x)}{x}\right)^{s}
$$

holds true for $0<|x|<a_{p}$ provided the numbers $\alpha, \beta, r$ and $s$ satisfy the following conditions

$$
s>0 \quad \text { and } \quad r \alpha \leq s \beta .
$$

Proof. Let

$$
u=\frac{g d_{p}(x)}{x} \quad \text { and } \quad v=\frac{g d_{p}^{-1}(x)}{x} .
$$

It is easy to see that both $u$ and $v$ are even functions of $x$. Thus without loss of generality we may assume that $x \in\left(0, a_{p}\right)$. Firstly we shall prove that $u$ and $v$ satisfy the separation condition

$$
u<1<v \text {. }
$$

In order to prove that $u<1$ we introduce the function $g(x)=x-g d_{p}(x)$. Differentiation yields

$$
g^{\prime}(x)=1-\left(\cosh _{p} x\right)^{1-p}>0,
$$

where the last inequality is a consequence of the fact that $\cosh _{p} x>1$. This in conjunction with $g(0)=0$ implies that $g(x)>0$ or what is the same that $u<1$. For the proof of the inequality $v>1$ we introduce the function $h(x)=x-g d_{p}^{-1}(x)$. Since

$$
h^{\prime}(x)=1-\left(\cos _{p} x\right)^{1-p}<0 \quad \text { and } \quad h(0)=0
$$

the assertion follows. Next we shall demonstrate that

$$
1<u v .
$$

Let $f(x)=g d_{p}(x)$ and let $\varphi(x):=\frac{f^{\prime}(x)}{x^{\prime}}$. Differentiation yields

$$
\varphi(x)=g d_{p}^{\prime}(x)=\frac{1}{\left(\cosh _{p} x\right)^{p-1}} .
$$


Thus the function $\varphi(x)$ is strictly decreasing on the interval $D=\left(0, a_{p}\right)$. Using Theorem 2 we conclude that the function $f(x) / x$ is also strictly decreasing on $D$. On the other hand, function $f^{-1}(x)=g d_{p}^{-1}(x)$ is strictly increasing on $D$. Finally, in order to show that $f(x)<x$ holds for all $x \in D$ it suffices to use 29$)$ and the inequality $1 /\left(\cosh _{p} x\right)^{p-1}<1$. Application of Theorem 1 completes the proof of the inequality 45). To finish the proof of this theorem we appeal to Theorem 3 with $\gamma=\delta=1$. Inequalities (24) and 25) imply, respectively (42) and (43).

Our next result reads as follows:

Theorem 7. If the numbers $r$ and s satisfy

$$
s>0 \quad \text { and } \quad r \alpha p \leq s \beta
$$

or if

$$
p \leq 2, \quad r \leq s \leq-1 \quad \text { and } \quad \beta \leq p \alpha,
$$

then the following inequality

$$
\alpha+\beta<\alpha\left(\frac{\sin _{p} x}{g d_{p}^{-1}(x)}\right)^{r}+\beta\left(\frac{\tan _{p} x}{g d_{p}^{-1}(x)}\right)^{s}
$$

holds true for all $0<|x|<a_{p}$.

Proof. Let $a$ and $b$ be positive numbers such that $a<b$. This implies the two-sided inequality

$$
a<S B_{p}(b, a)<b
$$

Define

$$
u=\frac{S B_{p}(b, a)}{b}, \quad v=\frac{S B_{p}(b, a)}{a} .
$$

This in conjunction with 49 yields

$$
u<1<v .
$$

We shall demonstrate now that

$$
1<u v^{p} .
$$

To this aim we utilize inequality 20

$$
a^{1 /(p+1)} b^{p /(p+1)}<\left[S B_{p}(b, a)\right]^{1 / p} b^{(p-1) / p} .
$$


An easy algebra yields (51). In order to demonstrate that (48) holds true if (46) is satisfied we employ Theorem 3 with $\gamma=1$ and $\delta=p$ and next we apply inequalities (25). We shall prove now that (48) also holds true if conditions (47) are satisfied. Firstly we utilize (21) assuming that $p \leq 2$. This yields

$$
S B_{p}(b, a)<\frac{1}{p+1}(b+p a),
$$

provided $b>a>0$. With $a=\cos _{p} x$ and $b=1$ the last inequality implies the following one

$$
\frac{1}{S B_{p}\left(1, \cos _{p} x\right)}>\frac{p+1}{1+p \cos _{p} x} .
$$

Next we utilize (55) together with the last inequality to obtain

$$
\frac{1}{u}>(p+1) \frac{1}{1+p \cos _{p} x} \text { and } \frac{1}{v}>(p+1) \frac{\cos _{p} x}{1+p \cos _{p} x} .
$$

With $\gamma=1$ and $\delta=p$ we have

$$
\gamma \frac{1}{u}+\delta \frac{1}{v}>(p+1) \frac{1+p \cos _{p} x}{1+p \cos _{p} x}=p+1=\delta+\gamma .
$$

Thus the condition (iii) of Theorem 3 is satisfied and the proof is complete.

We are in the position to establish the following:

Theorem 8. Assume that the numbers $\alpha, \beta, r$ and $s$ satisfy two inequalities

$$
s>0 \quad \text { and } \quad r \alpha \leq s \beta p .
$$

Then the inequality

$$
\alpha+\beta<\alpha\left(\frac{\tanh _{p} x}{g d_{p}(x)}\right)^{r}+\beta\left(\frac{\sinh _{p} x}{g d_{p}(x)}\right)^{s}
$$

holds for all $x \in\left(0, c_{p}\right)$.

Proof. We will use a method which bears resemblance of that used in the proof of the previous theorem. Suppose that the numbers $a$ and $b$ are such that $0<b<a$. This implies

$$
b<S B_{p}(b, a)<a .
$$


Let us define now quantities $u$ and $v$ :

$$
u=\frac{S B_{p}(b, a)}{a}, \quad v=\frac{S B_{p}(b, a)}{b} .
$$

This in conjunction with (54) implies that

$$
u<1<v \text {. }
$$

Next, we shall prove that

$$
1<u^{p} v
$$

To this aim we employ the left-hand side inequality of 20 written now as

$$
1<\frac{S B_{p}(b, a)^{p+1}}{a^{p} b}
$$

Applying (55) we obtain immediately inequality (57). We let now $a=$ $=\cosh _{p} x$ and $b=1$. Then (55) together with (34) imply

$$
u=\frac{\tanh _{p} x}{g d_{p}(x)} \quad \text { and } \quad v=\frac{\sinh _{p} x}{g d_{p}(x)} .
$$

In order to complete the proof we apply again Theorem 3 with $\gamma=p$, $\delta=1$ and $u$ and $v$ as given in (58).

We shall establish now the last result of this section.

Theorem 9. Assume that $\alpha$ and $\beta$ are unequal positive numbers. If the numbers $\alpha, \beta, r$ and $s$ satisfy the following conditions

$$
s>0 \quad \text { and } \quad r \alpha(p-1) \leq s \beta,
$$

then the inequality

$$
\alpha+\beta<\alpha\left(\frac{g d_{p}(t)}{t}\right)^{r}+\beta\left(\frac{\sinh _{p} t}{t}\right)^{s}
$$

is valid for all $t \neq 0$. Moreover, the inequality

$$
\alpha+\beta<\alpha\left(\frac{t}{g d_{p}^{-1}(t)}\right)^{r}+\beta\left(\frac{\tan _{p} t}{g d_{p}^{-1}(t)}\right)^{s}
$$

holds true provided $0<|t|<a_{p}$. 
Proof. Let the numbers $a$ and $b$ be such that $b<a$. Using $(19)$ we have $b<S B_{p}(a, b)<S B_{p}(b, a)$. This yields

$$
\frac{S B_{p}(a, b)}{S B_{p}(b, a)}<1<\frac{S B_{p}(a, b)}{b} .
$$

Let $u$ stand for the first member of $(62)$ and let $v$ denote the third member of $(62)$. Thus 62 can be written as

$$
u<1<v .
$$

Next we shall use inequality 20 to obtain

$$
1<\frac{\left(S B_{p}(a, b)\right)^{1 / p}\left(S B_{p}(a, b)\right)^{1-1 / p}}{\left(S B_{p}(b, a)\right)^{1 / p} b^{1-1 / p}}=u^{1 / p} v^{1-1 / p} .
$$

This implies the inequality

$$
1<u v^{p-1}
$$

Thus we can use Theorem 3 with $\gamma=1$ and $\delta=p-1$. To complete the proof we let $a=\cosh _{p} t$ and $b=1$. Making use of (34) and (36) we obtain

$$
u=\frac{g d_{p}(t)}{t} \quad \text { and } \quad v=\frac{\sinh _{p} t}{t} .
$$

We use Theorem 3 to obtain (59) and (60). To derive inequality (61) we let now $a=1 / \cos _{p} t$ and $b=1$. Making use of (37) and (38) we obtain

$$
u=\frac{t}{g d_{p}^{-1}(t)} \quad \text { and } \quad v=\frac{\tan _{p} t}{g d_{p}^{-1}(t)} .
$$

This completes the proof.

Acknowledgment. The author is indebted to an anonymous referee for constructive criticism and valuable remarks which helped him to revise the first draft of this paper.

\section{References}

[1] Anderson G. D., Vamanamurthy M. K., Vuorinen M. Monotonicity rules in calculus. Amer. Math. Monthly, 2006, vol. 133, no. 9, pp. 805-816. 
[2] Baricz A., Bhayo B. A., Klén R. Convexity properties of generalized trigonometric and hyperbolic functions. Aequat. Math., 2013. DOI:10.1007/s00010-013-0222-x.

[3] Bhayo B. A., Vuorinen M. On generalized trigonometric functions with two parameters. J. Approx. Theory, 2012, vol. 164, no. 10, pp. 1415-1426.

[4] Bhayo B. A., Vuorinen M. Inequalities for eigenfunctions of the $p$ Laplacian. Probl. Anal. Issues Anal., 2013, vol. 2(20), no. 1, pp. 13-35. DOI: $10.15393 /$ j3.art.2013.2322.

[5] Edmunds D. E., Gurka P., Lang J. Properties of generalized trigonometric functions. J. Approx. Theory, 2012, vol. 164, pp. 47-56.

[6] Klén R., Vuorinen M., Zhang X. Inequalities for the generalized trigonometric and hyperbolic functions. J. Math. Anal. Appl., 2014, vol. 409, pp. 521529.

[7] Lindqvist P. Some remarkable sine and cosine functions. Ricerche di Matematica, 1995, vol. 44, no. 2, pp. 269-290.

[8] Neuman E. Inequalities for weighted sums of powers and their applications. Math. Inequal. Appl., 2012, vol. 15, no. 4, pp. 995-1005.

[9] Neuman E. Inequalities involving generalized trigonometric and generalized hyperbolic functions. J. Math. Inequal., 2014, vol. 8, no. 4, pp. 725-736.

[10] Neuman E. On the inequalities for generalized trigonometric functions. Int. J. Anal., 2014, vol. 2014, Article ID 319837, 5 p.

[11] Neuman E. On the p-version of the Schwab-Borchardt mean. Int. J. Math. Math. Sci., 2014, vol. 2014, Article ID 697643, 7 p.

[12] Neuman E. On the p-version of the Schwab-Borchardt mean II. Int. J. Math. Math. Sci., 2015, vol. 2015, Article ID 351742, 4 p.

[13] Neuman E. Inequalities for the generalized trigonometric, hyperbolic and Jacobian elliptic functions. J. Math. Inequal., 2015, vol. 9, no. 3, pp. 709726.

[14] Neuman E. Wilker and Huygens-type inequalities for Jacobian elliptic and theta functions. Integral Transforms Spec. Funct., 2014, vol. 25, no. 3, pp. 240-248.

[15] Neuman E. Wilker and Huygens - type inequalities involving Gudermannian and the inverse Gudermannian functions. Probl. Anal. Issues Anal., 2017, vol. 6(24), no. 1, pp. 46-57. DOI: 10.15393/j3.art2017.3770.

[16] Olver F. W. J., Lozier D. W., Boisvert R. F., Clark C. W. (Eds.) The NIST Handbook of Mathematical Functions. Cambridge Univ. Press, New York, 2010. 
Received January 12, 2018.

In revised form, March 26, 2018.

Accepted April 11, 2018.

Published online May 15, 2018.

Southern Illinois University and Mathematical Research Institute 144 Hawthorn Hollow, Carbondale IL 62903, USA

E-mail: edneuman76@gmail.com 